\title{
Uso de Storytelling para Apoiar a Gestão de Conhecimento no Desenvolvimento de Software
}

\author{
Tamara M. Mendes' ${ }^{1}$, Carlos A. M. Pietrobon ${ }^{1,2}$ \\ ${ }^{1}$ Instituto de Ciências Exatas e Informática - Pontifícia Universidade Católica de Minas \\ Gerais Caixa Postal 30.535-901 - Belo Horizonte - MG - Brasil \\ ${ }^{2}$ Departamento de Computação - DECOM - UFOP \\ tammends@gmail.com, camp9700@gmail.com
}

\begin{abstract}
This paper proposes the technique and a tool of storytelling to assist the knowledge management in software development companies. The goal is to develop and evaluate a software tool that lets you create, retrieve and manage stories using multimedia resources to manage knowledge of software processes and product development. This knowledge is useful in developing system and in future projects. The tests of the proposed tool were performed in requirements elicitation phase of three projects. The results showed that the stories allow extracting more requirements of the system than just with traditional interviews, and they can be used as a complement thereof.
\end{abstract}

Resumo. Este trabalho propõe a técnica e uma ferramenta de contar histórias para auxiliar a Gestão de Conhecimento em empresas de desenvolvimento de software. $O$ objetivo é desenvolver e avaliar uma ferramenta que permite criar, recuperar e gerenciar histórias utilizando recursos multimídia para gerir conhecimento de processos de software e do produto desenvolvido. Este conhecimento é útil no sistema em desenvolvimento e em futuros projetos. Os testes da ferramenta proposta foram realizados na fase de levantamento de requisitos de três projetos. Os resultados mostraram que as histórias permitem extrair mais requisitos do sistema do que apenas com as entrevistas tradicionais, e podem ser utilizadas como complemento destas.

\section{Introdução}

O desenvolvimento de software é uma atividade envolvendo muito conhecimento e é de grande importância compartilhar este conhecimento com a equipe, para que a mesma tenha um bom desempenho e desenvolva produtos de qualidade [Lemos e Souza 2008]. Porém, muitas vezes, a comunicação e difusão de informações entre a equipe são falhas [Ye 2005], o conhecimento é implícito e difícil de documentar, fazendo com que as empresas percam tempo com problemas que já foram resolvidos anteriormente, diminuindo a produtividade [Rus e Lindvall 2002].

Um dos problemas com relação à transferência de conhecimento é relativo à dificuldade de como documentá-lo e transmiti-lo de forma natural e eficiente. Pilhas de documentos escritos, embora possam registrar muito conhecimento, não são utilizados na prática devido à dificuldade de fazê-los e de recuperar informação relevante deles. 
O conhecimento tem sido utilizado desde a pré-história para apoiar as mais diversas atividades e a técnica de contar histórias, tem sido uma forma natural de transmitir este conhecimento.

Assim, este trabalho propõe uma abordagem baseada na aplicação da técnica de contar histórias para criar e gerenciar conhecimento no contexto de um projeto de desenvolvimento de software usando uma ferramenta que executa na web.

Na seção 2 é feito um estudo bibliográfico. As funcionalidades da ferramenta desenvolvida - HqAdmin, são descritas na seção 3. Na seção 4 são detalhados os testes feitos com o HqAdmin e os resultados obtidos são analisados. Finalmente na seção 5 são feitas as conclusões e indicados os trabalhos futuros.

\section{Gestão de Conhecimento e Storytelling}

\subsection{Gestão de Conhecimento}

As organizações precisam cada vez mais gerenciar e compartilhar conhecimento entre os membros da equipe [Perret, Borges e Santoro 2004], o que incentiva as empresas a adotarem métodos que auxiliem na aquisição, armazenamento e disseminação do conhecimento - conjunto de processos que define Gestão de Conhecimento. A Gestão de Conhecimento é uma disciplina emergente que visa tirar proveito do conhecimento agregado das pessoas, tomando uma visão mais abrangente da informação [Rus e Lindvall 2002], [Perret, Borges e Santoro 2004]. Ela considera o indivíduo como portador de conhecimento que pode ser compartilhado com outros na tentativa de melhorar a produtividade da equipe [Levy e Hazzan 2009].

O conhecimento organizacional está incorporado e espalhado na memória dos funcionários, nos sistemas, procedimentos e produtos da empresa. Grande parte deste conhecimento é tácito. Conhecimento tácito é o conhecimento implícito que está espalhado na mente das pessoas da organização na forma de memória, habilidades, experiência, educação, imaginação e criatividade [Valle et al. 2003]. É obtido em ambientes informais, como conversas de corredor, histórias, notas rabiscadas em guardanapos e e-mails informais [Perret, Borges e Santoro 2004]. Ele é altamente pessoal e difícil de formalizar [Ajila e Sun 2004], [Levy e Hazzan 2009].

\subsection{Conhecimento e Software}

Software é conhecimento incorporado. Tal conhecimento está inicialmente disperso, tácito e incompleto, portanto, o desenvolvimento de software é um processo interativo de aprendizagem e o resultado é a incorporação de conhecimentos coletados, destilados e organizados [Pressman 2006].

No entanto, a realidade é que as equipes de desenvolvimento de software não reutilizam este conjunto de conhecimentos. Elas não se beneficiam de experiências anteriores e repetem os mesmos erros que outros membros já enfrentaram e sabem como evitá-los, atrasando os projetos [Rus e Lindvall 2002].

Se uma base de conhecimento, uma memória organizacional - registro de dados úteis, informação e conhecimento - não é implantada na empresa, todo conhecimento adquirido ao longo de um trabalho em grupo é perdido quando o projeto termina, 
quando o grupo se dissolve, ou quando um colaborador sai da empresa [Valle et al. 2003]. Novos membros da equipe levarão um tempo considerável para entender os projetos e os processos de trabalho, precisando da ajuda de outros membros da empresa, o que causa mais atrasos [Rus e Lindvall 2002].

Além disto, nas empresas de desenvolvimento de software, as fontes de conhecimento sofrem modificações constantemente, evoluindo com as mudanças tecnológicas, com a criação de novos conhecimentos, entre outros. Devido a isto, a base de conhecimento sempre estará incompleta [Ajila e Sun 2004], [Rus e Lindvall 2002].

A Gestão de Conhecimento tem tal importância que é exigida no MPS.BR no nível de maturidade E. Outros modelos de qualidade, como CMMI, também já consideram o tratamento do conhecimento em suas propostas. No caso do MPS.BR, os resultados esperados são: o estabelecimento de uma estratégia de gerência de conhecimento para compartilhar informações na organização; e a disponibilização e compartilhamento do conhecimento na organização que segue o modelo [SOFTEX 2010].

\subsection{Storytelling}

Storytelling - contar histórias - é uma prática muito antiga. Os Egípcios contavam histórias através de imagens. Depois da invenção da escrita, as histórias ficaram mais fáceis de serem registradas e diversas civilizações o fizeram.

Narração vem do latim narrere que significa "transmitir conhecimento" [Valle, Prinz e Borges 2002]. A narrativa é então um mecanismo para transmitir conhecimento. Uma habilidade antiga que está sendo aplicada a novos contextos, como o de Gestão de Conhecimento [Valle et al. 2003].

Contar histórias é uma maneira natural de transferir conhecimento, onde os stakeholders ${ }^{1}$ podem se expressar sem as limitações da linguagem de relatórios formais, fazendo uma releitura de eventos que ocorreram no passado [Santoro, Borges e Pino 2010]. Podem também descrever situações de um contexto indicando diferentes visões de um mesmo processo. E podem contar histórias em grupo, ou seja, mais de uma pessoa pode participar do processo de contar histórias, tornando-a mais rica [Valle et al. 2003], [Luz, Borges e Campos 2008].

Contar histórias é uma prática tão popular e importante que vem sendo aplicada em diversos campos [Santoro, Borges e Pino 2010]. Como na área de Gestão de Conhecimento [Valle, Prinz e Borges 2002], [Valle et al. 2003], [Perret, Borges e Santoro 2004]. É usada como ferramenta de ensino [Tsou, Wang e Tzeng 2003], [Kelleher, Pausch e Kiesler 2007], [Christensen 2009]. Também é aplicada para identificar inovações numa empresa [Escalfoni, Braganholo e Borges 2009]. Para modelagem de processos de uma organização [Santoro, Borges e Pino 2008], [Santoro, Baião e Gonçalves 2009] e [Santoro, Borges e Pino 2010]. Para apoiar levantamento de requisitos [Winbladh, Ziv e Richardson 2010].

\footnotetext{
${ }^{1}$ Pressman (2006) explica que um stakeholder é alguém interessado no sucesso do projeto. Podem ser engenheiros de software, gerentes, usuários finais, clientes, analistas, enfim, qualquer pessoa envolvida no processo.
} 
Devido a tantas aplicações foi desenvolvido um framework - SOFIA, para ser usado no desenvolvimento de ferramentas de apoio a storytelling em grupo [Luz, Borges e Campos 2008].

Um dos maiores benefícios de contar histórias é sua natureza dinâmica. É uma maneira mais simples e natural de descrever e compartilhar experiências do que muitas tecnologias complexas disponíveis nas áreas de Gestão de Conhecimento [Valle et al. 2003]. Mas, infelizmente, se contar histórias é uma atividade muito realizada informalmente, não o é formalmente, entre outros motivos por causa da dificuldade em se ter meios fáceis de registrar e recuperar o conhecimento.

\section{HqAdmin}

HqAdmin, é uma ferramenta que permite a construção, recuperação e o gerenciamento de histórias utilizando recursos multimídia, e que executa na web. Os casos de uso que expressam as funcionalidades da ferramenta podem ser vistos na Figura 1.

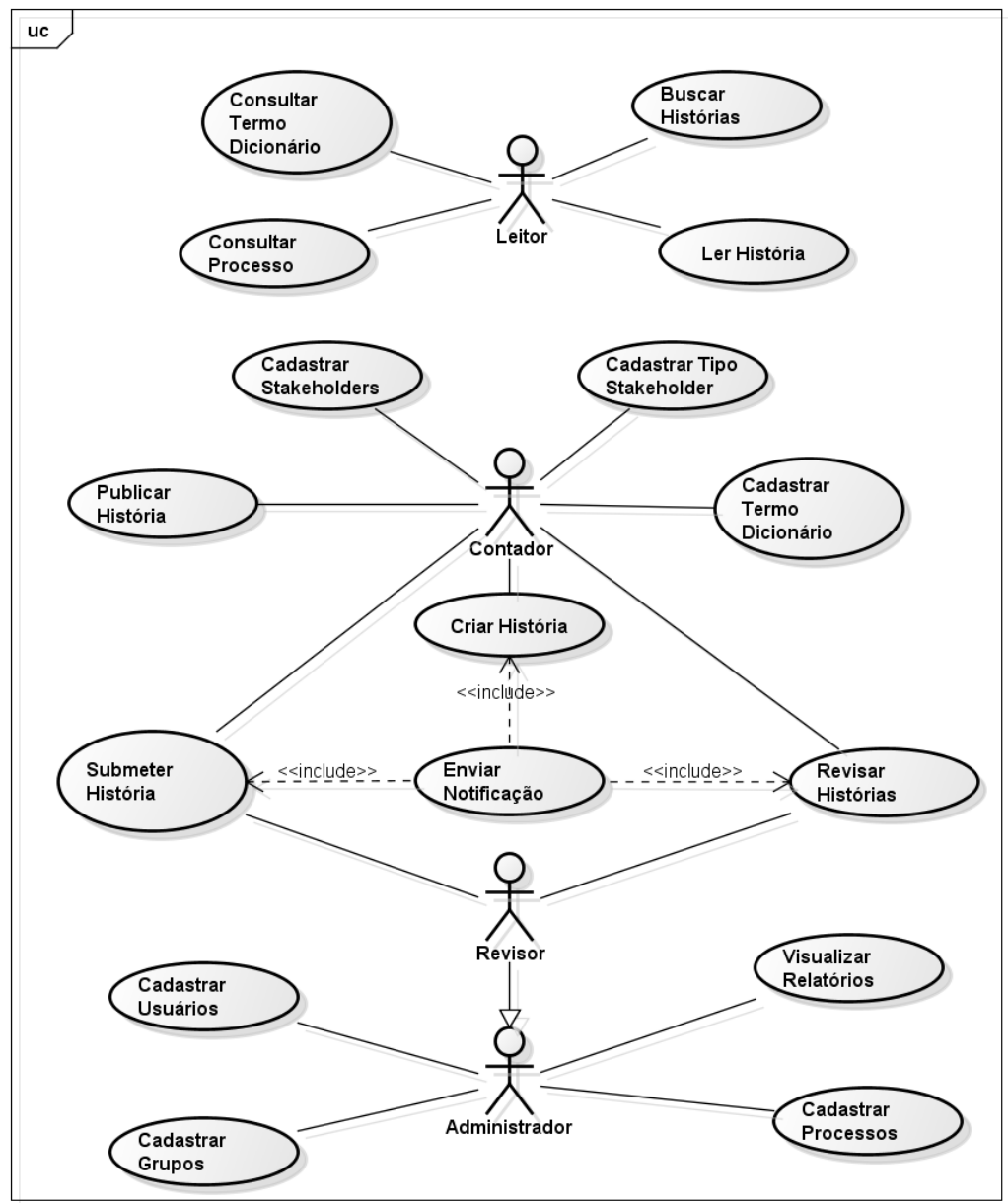

Figura 1. Diagrama de Casos de Uso

A ferramenta web foi construída usando a linguagem Java na versão 7 e o framework JSF na versão 2.0. O banco de dados utilizado foi o MySQL. Os módulos de construção e recuperação de histórias foram feitos em Applet Java que permite a 
construção de uma interface mais dinâmica usando arquivos multimídia, e ao mesmo tempo, a integração às páginas web.

O módulo principal do sistema é o de construção das histórias. Ele permite criar histórias em formato de eventos usando recursos multimídia. Há o componente que exibe uma história para leitura, outro onde o revisor lê a história e revisa, e um componente para os autores da história lerem a revisão. Estes módulos foram acoplados à ferramenta web formando o HqAdmin.

\subsection{Processo de construção das histórias}

As histórias são compostas por uma sequência de eventos. Um evento é um quadrinho, como em HQ - História em Quadrinho, que pode conter uma combinação de texto, imagem, vídeo ou áudio, proposta encontrada em [Valle et al. 2003]. A Figura 2 mostra uma tela da ferramenta onde a história é criada.

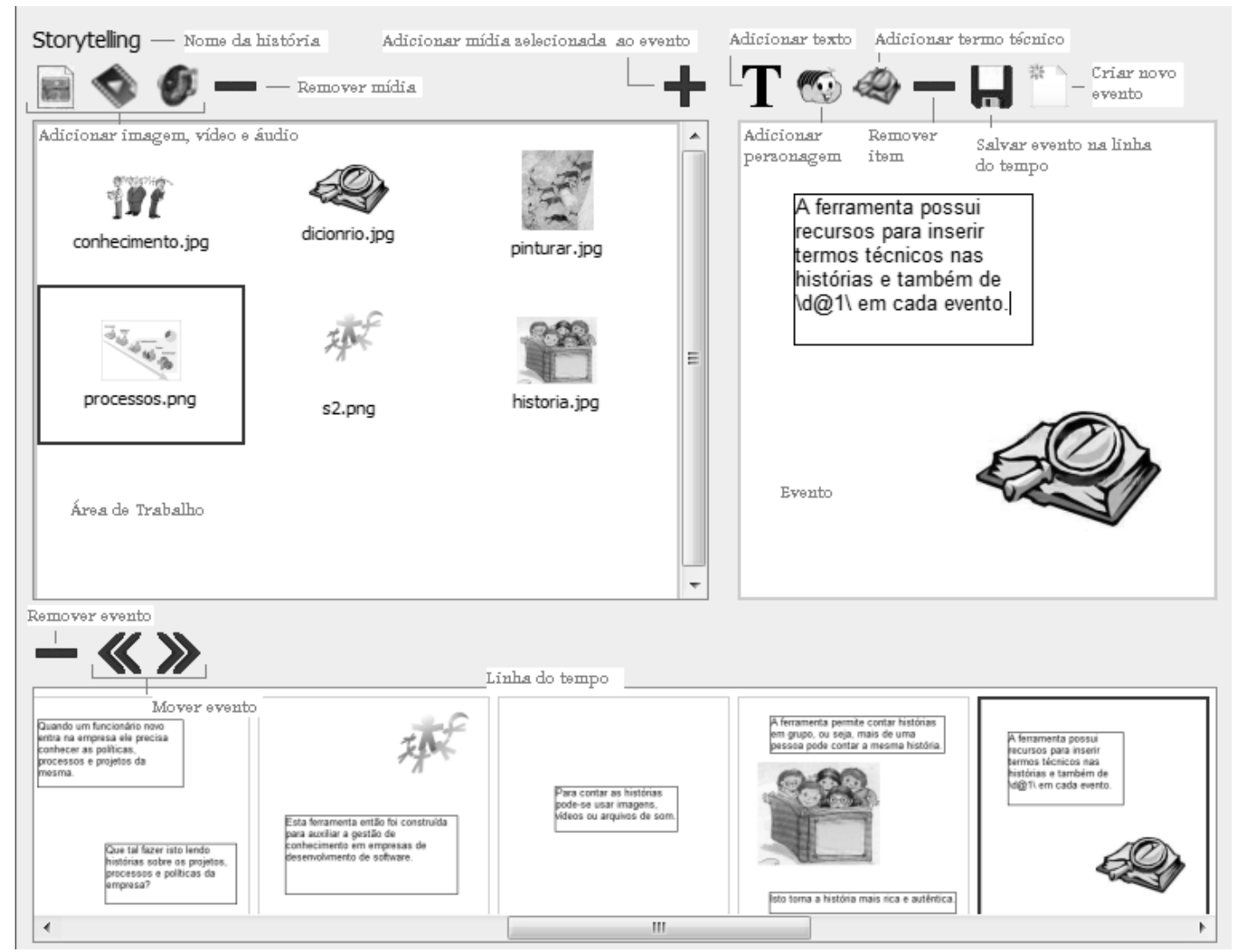

Figura 2. Módulo de criar história

As imagens e caixas de texto podem ser redimensionadas e reposicionadas dentro do evento. Os arquivos de áudio e vídeo podem ser arrastados e posicionados onde o contador da história preferir. Ao salvar um evento, ele é associado à história em construção e adicionado à linha do tempo - sequência em que os eventos são apresentados. Quando a história for lida, ao clicar no ícone do arquivo de mídia, ele será executado com o programa padrão instalado na máquina do usuário para aquele formato 
de arquivo. Na linha do tempo é possível excluir, editar e reposicionar um evento. Estrutura semelhante a esta é apresentada por [Santoro, Borges e Pino 2010].

Uma história pode ser contada por mais de uma pessoa, o que torna a história mais rica e autêntica [Luz, Borges e Campos 2008]. O autor principal é aquele que cria a história. Ele pode convidar autores secundários para participar da construção da mesma. Cada autor cria seus eventos. Os autores secundários não têm permissão para editar eventos criados por outros autores, podem apenas visualizá-los. Apenas o autor principal pode editar qualquer evento da história.

Quando finalizada, a história deve ser revisada, para então poder ser publicada, como foi sugerido em [Valle, Prinz e Borges 2002]. Para isto a história deve ser submetida a um revisor. Ele verificará se a história está dentro dos padrões aceitos e pode deferir ou indeferir a publicação da mesma. $\mathrm{O}$ revisor pode inserir comentários a respeito de cada evento para que os autores saibam o que corrigir. Caso seja indeferida, a história deverá ser corrigida para uma nova revisão. Quando a história for aceita pelo revisor, o autor principal pode publicá-la. A história não pode ser editada quando é submetida ao revisor, nem depois de publicada. Depois de enviada ao revisor, só poderá - e deverá - ser editada, se a história for indeferida pelo revisor.

A Figura 3 mostra uma tela da ferramenta onde a história é revisada. No evento exibido é mostrado um uso do dicionário técnico. O termo "Taleta" foi cadastrado no dicionário. Ao clicar em "Taleta", seu significado é exibido ao lado.

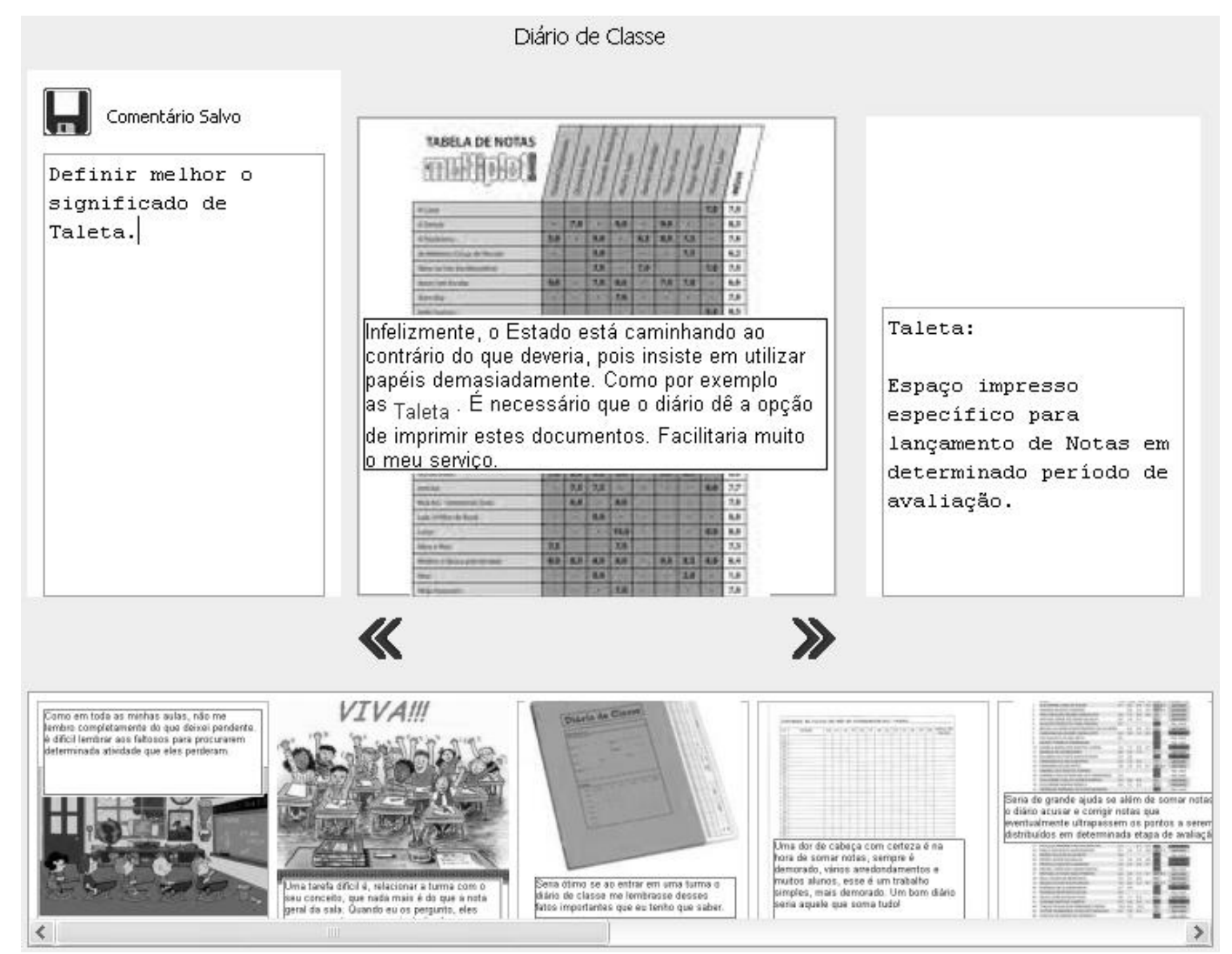

Figura 3. Módulo de revisar história 


\subsection{Outras funcionalidades da ferramenta}

Para recuperar as histórias foi feito um mecanismo de buscas por palavras-chave, proposto em [Valle, Prinz e Borges 2002]. As histórias também podem ser filtradas por processo, projeto ou por autor.

É possível fazer o cadastro de stakeholders. A eles são associados nome, imagem e cargos ou papéis executados pelos mesmos. O cadastro de stakeholders é feito para que possam ser referenciados nas histórias. Ao criar cada evento, podem ser incluídos personagens que fazem parte da história. Tais personagens são os stakeholders, que estão envolvidos de alguma forma na história. Quando a mesma for lida, haverá um link no nome do stakeholder que, ao abrir, exibe detalhes sobre o mesmo. A inserção de personagens foi apresentada nos trabalhos [Perret, Borges e Santoro 2004], [Kelleher, Pausch e Kiesler 2007].

Os stakeholders não são associados aos usuários do sistema. Isto porque um personagem pode não ser usuário do sistema. Pode ser um fornecedor, um cliente, ou mesmo um membro da empresa que não usa a ferramenta. Por outro lado, os usuários do sistema não são necessariamente membros da empresa. Um cliente, por exemplo, pode contar histórias sobre seu trabalho para que possam ser definidos melhor os requisitos do sistema a ser desenvolvido, já que é um processo envolvendo coleta de muito conhecimento sobre áreas que, muitas vezes, não são da alçada dos desenvolvedores [Kang et al. 2008].

A ferramenta também disponibiliza um dicionário técnico. Nele podem ser cadastrados termos ou palavras com seus respectivos significados. Ao escrever uma história os termos podem ser importados diretamente no texto. Quando a história for lida, os termos serão exibidos como links. Ao abrir, o significado do termo será exibido.

A ferramenta permite cadastrar projetos para associação de uma história ao projeto do qual ela faz parte, se faz parte de algum.

Ao criar uma história e selecionar os autores secundários e o revisor, notificações são enviadas a cada um deles, como foi proposto em [Valle et al. 2003]. As mensagens são enviadas para a caixa de mensagens do sistema e para o e-mail cadastrado do usuário.

Permissões de leitura devem ser dadas para cada história criada. Serão indicados grupos que tenham permissão para ler as histórias e pelo menos um grupo deverá ser indicado por história. Os grupos serão cadastrados previamente por um usuário que tenha permissão para administrar grupos. Assim, uma história não poderá ser restrita a leitores específicos que o autor principal escolher. Mas sim para grupos específicos. Isto evita que as fontes de conhecimento sejam privadas.

\subsection{Processo de software na ferramenta}

Um Processo de Software é um roteiro com uma série de passos para a elaboração de um produto ou sistema com qualidade e respeitando prazos [Pressman 2006]. Não obstante, em muitas organizações os processos não são bem documentados. Em algumas, eles nem são definidos [Santoro, Borges e Pino 2010]. Isto dificulta o entendimento e o reuso. 
Com o HqAdmin um processo pode ser definido e explicado por histórias. $\mathrm{O}$ processo possui atores, papéis - personagens da história, e seus respectivos comportamentos, atividades e tarefas [Santoro, Borges e Pino 2010]. O uso de histórias é uma forma mais simples e natural de documentar o conhecimento sobre o processo, tanto para quem escreve, como para quem lê. Principalmente para usuários que não têm treinamento, como o cliente, por exemplo [Santoro, Borges e Pino 2008]. Histórias são uma boa alternativa para explicar mais naturalmente porque um processo tem certa composição, o motivo da alocação de determinados colaboradores, os problemas que surgiram e as ações corretivas, dentre outros.

A documentação de um processo é o resultado de muitas atividades e decisões baseadas em conhecimento. Ela não registra estas decisões, problemas e alternativas de solução consideradas para se chegar ao resultado. Assim, acredita-se que as histórias sejam uma alternativa para explicar melhor estas informações que a documentação formal não explicita, não explica ou tem dificuldade para transmitir.

O sistema proposto, no seu estágio atual, permite o cadastro de processos para que possam ser contadas histórias sobre eles. Já que um Processo de Software está sempre em evolução, sendo melhorado com as experiências diárias, cada dia pode ser contada uma ou mais histórias sobre ele. As histórias são fontes para coletar experiências relacionadas ao processo. Assim, um leitor que precisa aprender sobre determinado processo pode ler histórias relacionadas a tal processo ou parte dele.

\subsection{Ferramentas semelhantes}

Ferramentas semelhantes à desenvolvida neste trabalho incluem Tellstory [Perret, Borges e Santoro 2004], uma ferramenta para a construção de histórias colaborativas. Ela permite que os usuários assumam papéis de contador, organizador, associador ou indexador na criação da história. Ela permite a edição, inclusão, união, exclusão e fragmentação dos eventos que compõem a história. Permite definir e editar personagens. Os usuários podem discutir através de um fórum e tomar decisões por meio de votação.

Outra ferramenta é o iMuse [Winbladh, Ziv e Richardson 2010], uma ferramenta web gráfica que pode ser usada por técnicos e não técnicos para expressar, manipular e visualizar requisitos em forma de narrativa. Porém as histórias devem ser narradas de maneira mais sistemática e formal de acordo com semânticas pré-definidas.

\section{Estudos de Caso}

Um problema da Gestão de Conhecimento é que os benefícios de sua aplicação podem levar algum tempo antes de aparecer [Rus e Lindvall 2002]. Por este motivo o processo de levantamento de requisitos foi tomado como base para os estudos de caso da ferramenta desenvolvida. Histórias foram usadas para capturar os requisitos. Assim, foi possível ter um resultado mais imediato da aplicação da ferramenta de auxílio à Gestão de Conhecimento.

No processo de levantamento de requisitos o cliente fornece informações sobre o objetivo do sistema ou produto, quais necessidades do negócio o mesmo deve satisfazer e como ele será usado. Existem vários problemas neste processo. Muitas vezes, o cliente não sabe o que é necessário no sistema, omite informações e não tem domínio completo do problema [Pressman 2006]. Isto porque o conhecimento sobre o negócio é tácito e 
está espalhado pelos diversos membros da organização, o que torna difícil a tarefa de capturar e formalizá-lo [Ajila e Sun 2004].

O levantamento de requisitos é apontado como a etapa mais difícil e importante do processo de desenvolvimento de software. É uma fase que envolve uma ampla gama de conhecimento, importante para que as funcionalidades do software sejam criadas corretamente e se evite erros e mudanças no futuro [Kang et al. 2008].

\subsection{Método}

O processo para cada teste envolveu as seguintes etapas:

1. Um analista $A$ entrevista o cliente interessado em obter um software e que tem conhecimento sobre o negócio. O cliente pode ser uma ou mais pessoas envolvidas. Nesta entrevista é levantada uma lista de requisitos inicial A;

2. O mesmo cliente que participou da entrevista utiliza a ferramenta para contar uma história sobre seu dia a dia no trabalho ressaltando os problemas que o software poderia solucionar. Tal história pode ser contada por mais pessoas, ou seja, se mais de um cliente estiver envolvido eles podem contar a história em grupo;

3. Um analista $B$, que não teve acesso à lista de requisitos levantada, analisa a história contada pelo cliente e tenta extrair os requisitos da história, montando uma lista de requisitos $\mathrm{B}$;

4. Os analistas $A$ e $B$ se reúnem para discutir e incorporar as listas em uma lista de requisitos $\mathrm{C}$.

\subsection{Testes}

Foram realizados três testes com projetos na fase de levantamento de requisitos:

1. Projeto de um sistema de gerenciamento e vendas em uma gráfica: foram levantados 14 requisitos na lista $\mathrm{A}$. Alguns deles se repetiram na lista $\mathrm{B}$, onde foram listados 13 requisitos e um deles foi melhor detalhado em 24 passos. Obteve-se então, na lista C, 22 requisitos, onde 2 deles foram melhor detalhados em relação à lista $\mathrm{A}$ e 8 deles foram novos. A história foi construída usando apenas elementos textuais. Foi utilizado o recurso de inserção de personagem na história. O usuário levou cerca de 30 minutos para desenvolver a história;

2. Projeto de um diário de classe eletrônico para uso de professores de escolas públicas: foram levantados 19 requisitos na lista $\mathrm{A}$ e 17 requisitos na lista $\mathrm{B}$. Destes, 14 foram novos e 2 foram melhor definidos, logo a lista de requisitos $\mathrm{C}$ foi montada com 33 requisitos. Foram utilizados textos e imagens para compor a história e recursos do dicionário técnico. O usuário levou cerca de 20 minutos para desenvolver a história;

3. Projeto de site para campanha de um vereador: na lista $\mathrm{A}$ foram levantados 11 requisitos. Já na lista $\mathrm{B}$, obteve-se 13 requisitos. Na lista $\mathrm{C}$ foram registrados 14 requisitos, onde 3 eram novos e 1 foi melhor definido em relação à lista $\mathrm{A}$. $\mathrm{A}$ construção da história foi feita usando-se textos e imagens em forma de diálogo entre personagens. O usuário levou cerca de 30 minutos para desenvolvê-la. 
Os analistas A e B que participaram dos testes foram alunos formandos do curso de Ciência da Computação da PUC Minas. O analista A realizou as três entrevistas e o analista $\mathrm{B}$ explorou as três histórias sem ter acesso à entrevista e à lista de requisitos levantada na mesma, ou seja, sem ter conhecimento sobre o negócio. Isto foi feito para que o conhecimento sobre o negócio não influenciasse na interpretação das histórias.

O usuário que participou do teste no projeto do sistema da gráfica foi um funcionário da mesma. $\mathrm{O}$ sistema estava em desenvolvimento quando os testes foram realizados. O projeto do diário de classe eletrônico não existe de fato, mas pode ser desenvolvido futuramente. $\mathrm{O}$ usuário entrevistado foi um professor que sente necessidade de um sistema do tipo. O site do vereador foi desenvolvido e a lista de requisitos levantada foi usada como documentação para o projeto. $\mathrm{O}$ entrevistado foi o analista de negócio.

Os usuários receberam uma rápida explicação, de poucos minutos, sobre as funcionalidades da ferramenta. Eles foram orientados a se expressar livremente na construção das histórias, usando todos os recursos que julgassem necessários.

A final da montagem das três listas de cada um dos sistemas, os requisitos foram validados pelos mesmos entrevistados. Os dados mostrados neste trabalho se referem aos requisitos aprovados. Eles foram coletados apenas na fase inicial do levantamento de requisitos dos projetos. Não houve nenhuma avaliação posterior.

\subsection{Resultados}

A Figura 4 mostra o gráfico dos resultados.

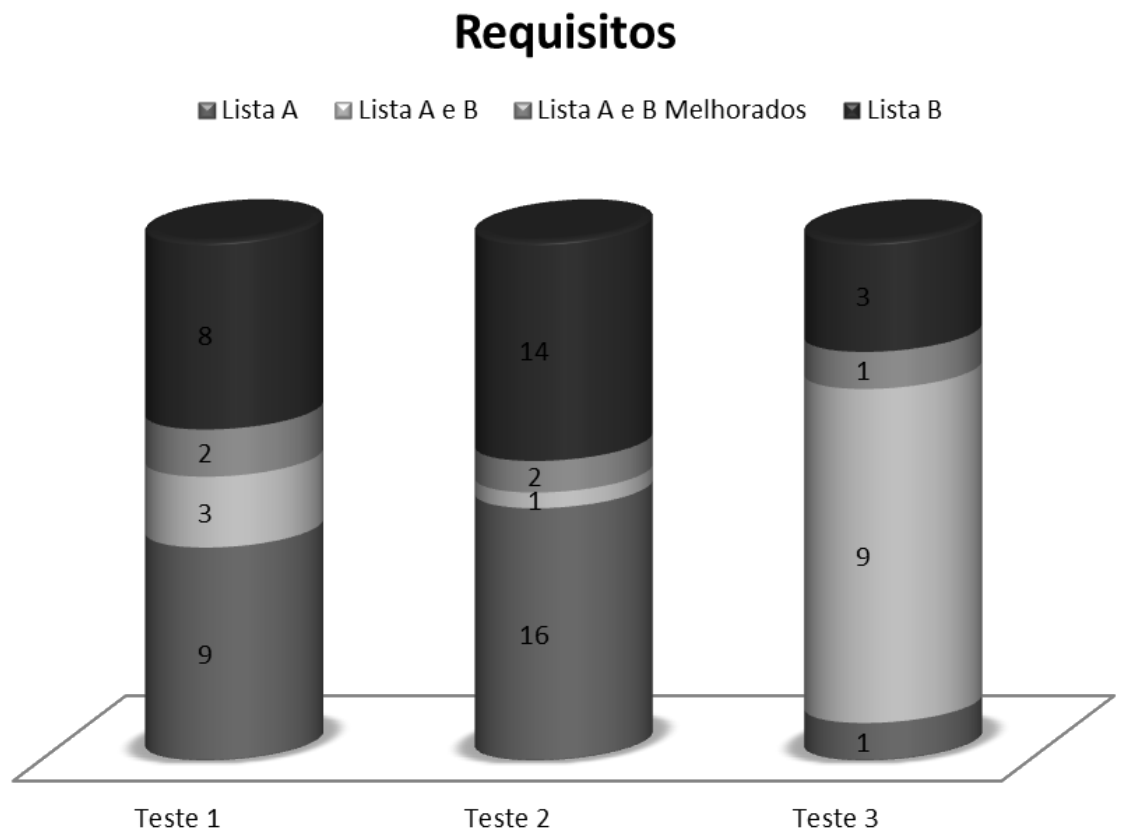

Figura 4. Requisitos da Lista C

Em cada coluna da Figura 4 são mostrados os requisitos da lista $\mathrm{C}$ que foi montada com os requisitos das listas A e B. A primeira camada, indicada na legenda 
como "Lista A", representa o número de requisitos levantados exclusivamente com a entrevista. As segunda e terceira camadas, apontadas na legenda respectivamente como "Lista A e B" e "Lista A e B melhorados", mostram o número de requisitos coletados tanto na entrevista como na história, entretanto, a terceira camada indica quantos destes requisitos foram melhor definidos com a análise da história. A última camada, indicada na legenda como "Lista B", aponta o número de requisitos levantados unicamente com a história.

Observa-se que através das histórias foi possível levantar um número maior de requisitos do que somente com a entrevista. Os requisitos levantados exclusivamente na lista B correspondem a $36 \%, 42 \%$ e $21 \%$ dos requisitos respectivamente nos testes 1,2 e 3 , que são consideradas porcentagens altas. O número de requisitos que foram melhorados com a história, ou seja, melhor definidos e compreendidos, correspondem respectivamente a $9 \%, 6 \%$ e $7 \%$ nos testes 1,2 e 3 . Estes números são bons resultados, já que o número de requisitos pode ser uma medida do entendimento do sistema a ser desenvolvido e quanto melhor for este entendimento nesta etapa do processo, melhor será o produto final e menor será o tempo de desenvolvimento, já que mudanças no projeto são mais difíceis se não forem definidas no início do desenvolvimento.

Os usuários do sistema levaram um tempo consideravelmente pequeno para construir as histórias. Isto indica que a ferramenta construída não exige muito tempo de dedicação por parte dos usuários, principalmente por ser uma técnica simples e natural. A eficiência é importante porque não é interessante que os colaboradores de uma empresa gastem mais tempo registrando conhecimento do que produzindo. Uma boa solução é aquela que não sobrecarregue os usuários, pois as empresas precisam ser mais eficientes e mais produtivas.

Em questionário feito com os usuários do sistema observou-se que alguns usuários sentiram falta de um recurso para inserir outros tipos de documentos nas histórias que não fossem arquivos multimídia.

\section{Conclusão}

A necessidade de implantação de Gestão de Conhecimento nas empresas surge com o grande volume de informação com o qual elas trabalham em conjunto com muitas pessoas. A empresa que gere conhecimento é melhor capaz de inovar, de não repetir erros do passado e de aumentar a produtividade e a competitividade. Porém esta implantação não é simples e pode exigir uma grande mudança cultural na empresa. Contar histórias é uma forma simples de capturar e documentar o conhecimento tácito e explícito. Desta forma, uma ferramenta para criar, recuperar e gerenciar histórias pode ser um passo inicial para implantação de Gestão de Conhecimento nas empresas.

Este trabalho propôs uma ferramenta para construção, recuperação e gestão de histórias no contexto de desenvolvimento de software, que serve para apoiar as atividades do processo, como visto no estudo de caso, bem como documentá-lo. Os testes realizados na fase de levantamento de requisitos de sistemas mostraram que storytelling, juntamente com a técnica usual de entrevistas, permite capturar mais e melhor os requisitos, já que o cliente tem mais liberdade para expressar o que pensa, seus problemas e o que precisa. Mais resultados podem ser obtidos futuramente com a 
utilização do sistema, pois os benefícios da aplicação de Gestão de Conhecimento podem levar algum tempo para aparecer.

O uso de recursos multimídia contribuiu para enriquecer e deixar as histórias mais interessantes e agradáveis, diferente dos relatórios formais geralmente usados nas empresas para registrar conhecimento.

As histórias construídas no processo de levantamento de requisitos já são conteúdo para a base de conhecimento da organização. Para exemplificar, em um possível projeto futuro, cujo negócio seja parecido com o registrado nas histórias, as mesmas poderão ser recuperadas para auxiliar no entendimento dos requisitos do novo projeto. Ou seja, as histórias deste processo além de ajudarem no levantamento de requisitos do projeto atual, também contribuem para Gestão de Conhecimento da empresa.

Com base nos estudos e testes realizados, conclui-se que storytelling é uma possível técnica para auxiliar a Gestão de Conhecimento em empresas de desenvolvimento de software de forma simples. Conclui-se também que uma ferramenta para criação, recuperação e gestão de histórias utilizando recursos multimídia é um instrumento para tornar a aplicação desta técnica possível. Permitindo gerir conhecimento do produto, como mostrado nos casos de teste, e dos processos de software.

Como trabalhos futuros, deseja-se usar a ferramenta para documentar o processo de software e avaliar seu uso e reuso em processos de projetos futuros. Pretende-se realizar mais testes explorando outras maneiras de avaliar a contribuição das histórias para levantamento de requisitos e para outras fases do desenvolvimento de software.

Também indicam-se melhorias e inserção de novas funcionalidades à ferramenta. Como exemplos, tem-se um recurso para que, ao escolher autores secundários para a história, os mesmos possam recusar ou aceitar o convite; a criação de um fórum; o aperfeiçoamento do dicionário técnico para uma ontologia; a possibilidade de importar processos diretamente de um arquivo; a pré-definição de temas para serem usados na construção das histórias; o reaproveitamento de uma história publicada para a criação de outra; e a migração dos módulos feitos em Applet para uma solução que possa ser usada em dispositivos móveis.

\section{Referências}

Ajila, S. and Sun, Z. (2004) "Knowledge management: impact of knowledge delivery factors on software product development efficiency", In: Proceedings of the IEEE International Conference on Information Reuse and Integration. Las Vegas, NV, United States: IEEE. p. 320-325.

Christensen, H. B. (2009) "A story-telling approach for a software engineering course design”. SIGCSE Bull., ACM, New York, NY, USA, v. 41, p. 60-64.

Escalfoni, R., Braganholo, V. and Borges, M. R. S. (2009) “Applying group storytelling to capture innovation features", In: Proceedings of the $200913^{\text {th }}$ International Conference on Computer Supported Cooperative Work in Design. Washington, DC, USA: IEEE Computer Society. p. 209-214. 
Kang, F., Fan, Z., Yu, H. and Cui, Y. (2008) "Research on construction of the knowledge system for software development", In: Proceedings of the 2008 International Symposium on Knowledge Acquisition and Modeling. Washington, DC, USA: IEEE Computer Society. p. 161-165.

Kelleher, C., Pausch, R. and Kiesler, S. (2007) "Storytelling alice motivates middle school girls to learn computer programming", In: Proceedings of the SIGCHI conference on Human factors in computing systems. New York, NY, USA: ACM. p. $1455-1464$.

Lemos, A. and Souza, C. de. (2008) "Desafios da gerência de conhecimento no desenvolvimento de software: Resultados de um estudo etnográfico", In:, Simpósio Brasileiro de Sistemas Colaborativos: IEEE Computer Society. p. 77-87.

Levy, M. and Hazzan, O. (2009) "Knowledge management in practice: The case of agile software development", In: Proceedings of the 2009 ICSE Workshop on Cooperative and Human Aspects on Software Engineering. Washington, DC, USA: IEEE Computer Society. p. 60-65.

Luz, C. M., Borges, M. R. and Campos, M. L. M. (2008) "Sofia: a framework for the development of group storytelling tools", In: Proceeding of the 2nd ACM international workshop on Story representation, mechanism and context. New York, NY, USA: ACM. p. 41-48.

Perret, R., Borges, M. and Santoro, F. (2004) “Applying group storytelling in knowledge management”, In: Vreede, G.-J. de; Guerrero, L.; Raventós, G. M. (Ed.). Groupware: Design, Implementation, and Use. San Carlos, Costa Rica: Springer Berlin/ Heidelberg, (Lecture Notes in Computer Science, v. 3198). p. 34-41.

Pressman, R. S. (2006) Engenharia de Software. $6^{\text {th }}$ edition. São Paulo: McGraw-Hill.

Rus, I. and Lindvall, M. (2002) "Knowledge management in software engineering", IEEE Software, v. 19, n. 3. p. 26-38.

Santoro, F. M., Baião, F. A. and Gonçalves, J. C. d. A. R. (2009) "Business process mining from group stories", In: Proceedings of the $200913^{\text {th }}$ International Conference on Computer Supported Cooperative Work in Design. Washington, DC, USA: IEEE Computer Society. p. 161-166.

Santoro, F. M., Borges, M. R. S. and Pino, J. A. (2008) "Tell us your process: A group storytelling approach to cooperative process modeling", In: Computer Supported Cooperative Work in Design. $12^{\text {th }}$ International Conference on. Rio de Janeiro, Brazil. p. 29-34.

Santoro, F. M., Borges, M. R. S. and Pino, J. A. (2010) "Acquiring knowledge on business processes from stakeholders' stories", Adv. Eng. Inform., Elsevier Science Publishers B. V., Amsterdam, The Netherlands, The Netherlands, v. 24. p. 138-148.

SOFTEX. (2011) "MPS.BR - Melhoria de Processo do Software Brasileiro. Guia Geral".

Tsou, W., Wang, W. and Tzeng, Y. (2003) "Applying computer multimedia storytelling website in foreign language learning", In: The 3rd IEEE International Conference on Advanced Learning Technologies. Proceedings. Taiwan: IEEE. p. 262-263. 
Valle, C., Prinz, W. and Borges, M. (2002) "Generation of group storytelling in postdecision implementation Process", In: The $7^{\text {th }}$ International Conference on Computer Supported Cooperative Work in Design. Rio de Janeiro, Brazil: IEEE. p. 361-367.

Valle, C., Raybourn, E. M., Prinz, W. and Borges, M. R. S. (2003) "Group storytelling to support tacit knowledge externalization", In: Proceedings of the $10^{\text {th }}$ International Conference on Human-Computer Interaction. Mahwah, New Jersey, USA: Lawrence Erlbaum Associates. p. 1218-1222.

Winbladh, K., Ziv, H. and Richardson, D. J. (2010) "Imuse: interactive model-based use-case and storytelling environment", In: Proceedings of the eighteenth ACM SIGSOFT international symposium on Foundations of software engineering. New York, NY, USA: ACM. p. 383-384.

Ye, Y. (2005) "Dimensions and forms of knowledge collaboration in software development", In: Proceedings of the $12^{\text {th }}$ Asia-Pacific Software Engineering Conference. Washington, DC, USA: IEEE Computer Society. p. 805-812. 